\title{
PULSE WAVE PROPAGATION ALONG HUMAN AORTA: A MODEL STUDY
}

\author{
NATALYA KizILOVA \\ Warsaw University of Technology, Institute of Aeronautics and Applied Mechanics, Warsaw, Poland, and \\ V.N. Karazin Kharkov National University, Kharkov, Ukraine \\ e-mail: n.kizilova@gmail.com \\ JEREMI MiZERSKI \\ Provincial Hospital in Zamość, Zamość, Poland \\ e-mail: jeremi.mizerski@gmail.com \\ Helen Solovyova \\ Kharkov National Technical University, Kharkov, Ukraine \\ e-mail: helenfilippova@yahoo.co.uk
}

\begin{abstract}
In the study, wave propagation along aorta is studied for different normal and pathological conditions in distal arteries. The mathematical model is based on the axisymmetric incompressible Navier-Stokes equations for blood and momentum equations for an incompressible viscoelastic arterial wall. The solution has been found as a superposition of forward and backward running waves. The blood pressure and flow curves measured by ultrasound in larger systemic arteries of ten healthy volunteers have been used for identification of the model parameters. It is shown that individual geometry plays an essential role in the location of positive and negative wave reflection sites along the aorta and, thus, in the pressure and flow patterns as well as blood distribution into the side branches. The model is validated by comparative study with the same dependencies computed previously on a 55-tube model as well as on the measurement data. The model can be used for determination of the individual parameters for patient-specific cardiovascular models and further in silico modeling of the outcomes of surgical and therapeutic procedures.
\end{abstract}

Keywords: applied mechanics, biomechanics, waves in fluids, wave reflection, medical diagnostics

\section{Introduction}

Models and methods of theoretical and applied mechanics form a basis for treatment and biomechanical interpretation of measured cardiovascular signals like blood pressure, velocity, flow rate, vessel wall oscillations and others (Nichols and O'Rourke, 2005). Significant progress in non-invasive measurement techniques like computed tomography (CT), magnetic resonance imaging (MRI), Doppler ultrasound (US), and whole body scanner (WBS) allows detailed 3D imaging of the blood circulation system up to medium arteries (CT, MRI, WBS), pressures and blood flows in larger arteries (US), 3D velocity profiles in larger (MRI) and medium (WBS) arteries, and integral blood flow and geometry of small vessels (micro CT) (Salvi, 2017). Detailed mathematical models based on fluid mechanics for blood flows and fluid-structure interaction (FSI) for vessel wall oscillations are needed to treat and interpret the measured data from the biomechanical point of view. The future technologies of medical diagnostics will be based on detailed computational results on patient-specific biomechanical models of blood circulation. The inverse problem solution on such models will allow determination of the most dangerous sites of wave 
reflections in the system that cause prolonged blood insufficiency in some organ(s), which leads to development of chronic diseases.

Aorta is the main blood vessel that directs, controls and distributes the blood flow into different parts of the body, internal organs and tissues. Small excitations produced by heart contractions propagate along the aorta and its branches at the speeds $c \sim 5-12 \mathrm{~m} / \mathrm{s}$, which exceeds the average linear blood flow velocity in the aorta $V \sim 50-80 \mathrm{~cm} / \mathrm{s}$ (Nichols and O'Rourke, 2005). Therefore, before the injected portion of blood reaches the distal part of the aorta, the propagating pulse wave reflects at each non-uniformity like aortic side branches, stenoses, aneurisms, atherosclerotic plaques in both downstream and upstream directions what significantly influences the flow rate, blood distribution and delivery (Salvi, 2017; Hughes et al., 2013). In medicine, there is a known phenomenon of "blood stealing" when one organ suffers of blood insufficiency while another receives more blood that is needed due to anomalous wave reflections and other reasons (Takach et al., 2006). The phenomenon was explained on the model of blood circulation as a hydraulic system, but later it was established that the wave components, especially the first harmonics delivered blood to the organs through aorta as a complex waveguide (Wang and Parker, 2004).

Individual geometry of the aorta and its local congenital or acquired abnormalities (Nichols and O'Rourke, 2005; Salvi, 2017; Hughes et al., 2013) are essential for development of complex diseases. For instance, a significant reflected wave originated at the aortic bifurcation may stimulate development of aneurism of abdominal aorta (Salvi, 2017; Hughes et al., 2013; Hughes et al., 2013; Takach et al., 2006). Wave reflections at the aortic narrowing or coarctation are dangerous for low blood delivery to the organs below the narrowed segment (Salvi, 2017; Hughes et al., 2013). Early wave reflections produced in rigid aorta cause direct damage to the aortic valve and development of systolic hypertension (Salvi, 2017; Hughes et al., 2013). Stenoses of the main coronary arteries produce wave reflection and insufficient blood delivery to the heart (Nichols and O'Rourke, 2005; Salvi, 2017).

Aortic flow is complex and not completely understood yet. The MRI studies detected appearance of vortex, helical, and reverse flows in aorta (Salvi, 2017; Hughes et al., 2013; Takach et al., 2006). Retrograde and rotational blood flows in the thoracic aorta were detected in all patients with systemic emboli and mobile protruding aortic atheroma, but general mechanisms of such flows have not been detected (Westerhof et al., 2006; Michail et al., 2018).

Since all internal organs are combined by the aorta into a complex biomechanical system, the properties of aorta as a waveguide are essential for deeper understanding of the diseases and the functional reasons of their development. The complex consideration of the human body is needed for advanced strategies of medical knowledge, diagnostic and treatment/surgery planning, which is the aim of the recently announced global "One Health Project" (Hall and Ba Le, 2015). The project emphasizes that medical diagnostics of a certain disease or local impairment is not a local diagnostic problem only, and needs a system dynamics approach to the entire vasculature. This paper is dedicated to further development of the most complete biomechanical model of human aorta that includes all known side branches with their detailed geometry. The model could be completed by an anomalous number and location of some side branches only. The model could be a basis for the one-health approach to medical diagnostics, treatment planning and monitoring as a digital technology for medicine.

\section{Biomechanical models of human aorta}

The very first model of aorta with its side branches was proposed in (Westerhof et al., 1969) based on the post-mortem measurements on an individual ( $175 \mathrm{~cm}$ height $75 \mathrm{~kg}$ mass). It contained 21 segments including 10 aortic segments and 11 side branching (Fig. 1) with diameters, lengths 
and wall thicknesses averaged on four datasets. It is a part of the well-established 55-tube model of the human systemic arterial tree. This model was validated on numerous in vivo data and found useful for general understanding of such mechanical phenomena as retrograde blood flow during diastole in some arteries (Wang and Parker, 2004), influence of individual anatomy of the radial and ulnar arteries on the blood flow in the upper extremities (Alastruey et al., 2006), structure of the circle of Willis on the blood distribution in the brain (Alastruey et al., 2007) and some others. In such problems, any computations on local models of arteries in brains or upper extremities is insufficient without accounting for aorta as the main pathway for the blood delivery. It was also shown that the 21-tube model of aorta corresponds to the so called "optimal waveguide" that provides almost zero wave reflections along it due to well-matched wave admittances at each side branching (Wang and Parker, 2004).

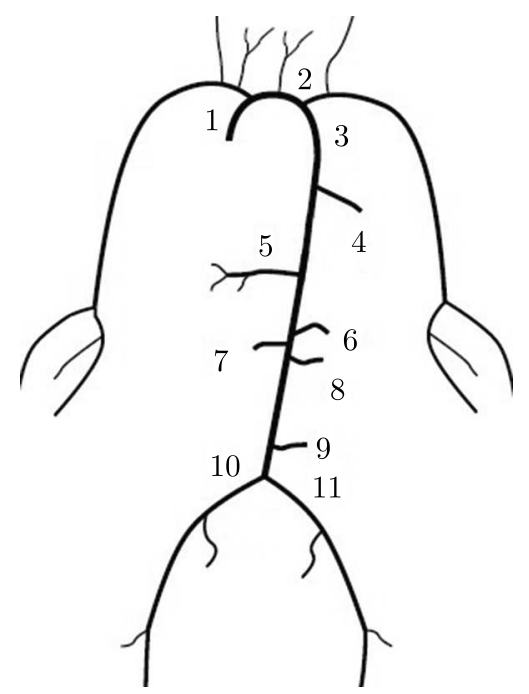

Fig. 1. The 55-tube model of human systemic arteries with the 21-tube model of aorta with: (1) brachiocephalic, (2) left carotid, (3) left subclavian, (4) intercostal, (5) ciliac, (6) left renal, (7) right renal, (8) upper mesenteric, (9) low mesenteric, (10) left iliac, (11) right iliac arteries

Later, the 55-tube model was completed to a 128-tube model, but the model of aorta has not been improved. Despite such a simplification, the 55-tube model exhibited good qualitative correspondence to in vivo data, but now much more detailed models are needed to treat terabytes of CT, MRI, US, WBS information and other available medical data. Besides, doctors need a more detailed description of their region of interest along the aorta. For instance, pulmonologists need the complete structure of the pulmonary, intercostal and subcostal arteries along the thoracic aorta to understand blood circulation in the respiratory system, while in the 21-tube model of aorta only one intercostal artery is present (Fig. 1). Nephrologists are interested in the complete structure of individual aorta that determines blood delivery to kidneys.

The biomechanical model of aorta as a waveguide composed by 93 tubes has been presented in our previous studies (Kizilova and Mizerski, 2018; Kizilova et al., 2019). As it was shown there, the aorta is an optimal waveguide not only due to well-matched branching with zero wave reflections. The most important is that along some regions of the thoracic aorta negative wave reflections appear which facilitates blood delivery to the abdominal aorta and low extremities due to the suction effect (Kizilova and Mizerski, 2018). In this paper, the influence of wave reflections on pulse wave propagation along the aorta is studied on the 93-tube model. The aim of the work is to study sensitivity of the model to individual geometry and material parameters of the vessels, and anomalous low or high resistivity of some side branches, which is essential for practical implementation of the model in medical diagnostics. 


\section{Review of mathematical models of aortic flow}

Over the past decade, the strong need of multi-scale models of the human circulation system for numerical simulations and computation-based approaches for medical diagnostics and decision making has been substantiated (Raphael et al., 2016). It was shown that the blood flow and wave propagation in large and medium arteries can be described on 1D (plug flow and plane waves) and 2D (axisymmetric flow and cylindrical waves) models, whereas small vessels and capillaries can be studied on 0D (lumped parameter) models treated as outlets boundary conditions for 1D and 2D models. The regions of specific interests like coronary arteries for cardiologists, kidneys for nephrologists, lungs for pulmonologists, and others can be computed on 3D FSI models embedded into the general simplified 1D/2D model of larger arteries (Quarteroni, 2001).

The problem of mathematically correct boundary conditions at the outlet of terminal tubes was discussed in (Quarteroni, 2001; Formaggia et al., 2001). It was shown that the 0D models can serve not only as terminal elements for 1D models (Nichols and O'Rourke, 2005; Formaggia et al., 2001) but can also substitute 1D elements for model simplification purposes (Quarteroni et al., 2000; Epstein et al., 2015). The synthetic 3D+1D, 3D+0D and 1D+0d models of aorta were worked out, and the influence of geometry and wall properties on $P(t)$ and $V(t)$ waveforms in aorta was studied (Quarteroni, 2001; Quarteroni et al., 2000; Epstein et al., 2015; Narayan et al., 2017; Negoita et al., 2017; Diaz-Zuccarini et al., 2006). In this Section, a brief review of the models used for computations on the 93-tube model of human aorta is given.

\subsection{The lumped parameter (OD) model}

The model considers a complex arterial network as a single elastic chamber with undisturbed volume $V_{0}$, compliance $C$ and peripheral resistivity $Z$ (Nichols and O'Rourke, 2005). The pressure oscillations $P(t)$ in the chamber are governed by the equation for pressure

$$
C \frac{d P}{d t}=Q_{\text {in }}(t)-\frac{P}{Z}
$$

where $Q_{\text {in }}(t)$ is the blood inflow into the chamber.

The model was formulated by Otto Frank in 1899 for the aorta as a single elastic chamber in which $Q_{\text {in }}(t)$ was the blood outflow from the left ventricle measured on the patient. For the 55 -tube model, the function $Q_{i n}(t)$ corresponds to the blood outflow from the corresponding open-end tube, and the $0 \mathrm{D}$ element models the inner organ. The typical parameters for 24 openend tubes of the 55-tube model can be found in a series of papers (Wang and Parker, 2004; Alastruey et al., 2006, 2007).

When the function $Q_{i n}(t)$ is known from the measurements, the solution to (3.1) is

$$
P(t)=\exp \left(-\frac{t}{Z C}\right)\left(P_{0}+\frac{1}{C} \int_{0}^{t} Q_{i n}(\tau) \exp \left(\frac{\tau}{Z C}\right) d \tau\right)
$$

where $P_{0}=P(0)$ is the diastolic pressure.

Equation (3.2) describes exponential pressure decay during the diastole when $Q_{i n}=0$ and allows determination of the model parameters $C$ and $Z$ from the measured $P(t)$ curves. Frank also modeled the aorta as a series connection of two elastic chambers with different resistive and compliant properties (thoracic and abdominal aorta) and studied relations between the pressures $P_{1}(t)$ and $P_{2}(t)$ in them, depending on their properties.

\subsection{D flow model (plane waves)}

The 1D model of blood flow in compliant tubes deals with a periodic flow $V(t, x), P(t, x)$ of an incompressible Newtonian fluid along a cylindrical tube of an arbitrary cross-section 
$\left.S(t, x)\right|_{t=0}=S_{0}(x)$ (undisturbed state) (Nichols and O'Rourke, 2005)

$$
\frac{\partial S}{\partial t}+\frac{\partial(V S)}{\partial x}=0 \quad \frac{\partial V}{\partial t}+V \frac{\partial V}{\partial x}=-\frac{1}{\rho_{f}} \frac{\partial P}{\partial x}+\frac{k \mu_{f} V}{\rho_{f} S}
$$

where $V$ is the axial velocity, $\rho_{f}$ and $\mu_{f}$ are density and viscosity of the fluid, $k$ is the friction coefficient at the wall.

According to the experimental data (Alastruey et al., 2006, 2007; Epstein et al., 2015), the rheological relation for the 1D model of the arterial wall can be approximated as

$$
P(S)=P_{0}+\frac{\beta}{S_{0}}\left(\sqrt{S}-\sqrt{S_{0}}\right) \quad \beta=\frac{4 \sqrt{\pi} E h}{3\left(1-\nu^{2}\right)}
$$

where $E, h, \nu$ are Young's modulus, thickness and Poisson's ratio for the arterial wall, $P_{0}=P\left(S_{0}\right)$.

Model (3.3)-(3.4) is very popular for blood flow computations in complex geometries of the human systemic arterial tree (Wang and Parker, 2004), brain arteries (Alastruey et al., 2007), upper and lower extremities (Alastruey et al., 2006, Karamanoglu et al., 1995). It could be reduced to model (3.1) by determining the resistivity and compliance of each tube in the geometrical model as

$$
Z=\frac{\rho_{f} c}{S_{0}} \quad C=\frac{1}{\rho_{f}} \int_{0}^{L} \frac{S_{0}(x)}{c_{0}^{2}} d x \quad c=\sqrt{\frac{\beta \sqrt{S}}{2 \rho_{f} S_{0}}}
$$

where $c$ is the pulse wave speed, $c_{0}=\left.c\right|_{S=S_{0}}, L$ is length of the tube.

The resistivity and compliance of arterial bifurcations (branches) can be computed as parallel and serial connections of single tubes (Epstein et al., 2015).

\subsection{Axisymmetric 2D flow (cylindrical waves)}

The model was developed by John R. Womersley in 1855 and was based on linearized incompressible Navier-Stokes equations for an axisymmetric pulsatile flow with blood velocity $\mathbf{v}(t, r, x)=\left[v_{r}, 0, v_{x}\right]$ and pressure $p(t, r, x)$

$$
\begin{aligned}
& \frac{1}{r} \frac{\partial\left(r v_{r}\right)}{\partial r}+\frac{\partial v_{x}}{\partial x}=0 \\
& \rho_{f} \frac{\partial v_{x}}{\partial t}=-\frac{\partial p}{\partial x}+\mu\left(\frac{1}{r} \frac{\partial}{\partial r}\left(r \frac{\partial v_{x}}{\partial r}\right)+\frac{\partial^{2} v_{x}}{\partial x^{2}}\right) \\
& \rho_{f} \frac{\partial v_{r}}{\partial t}=-\frac{\partial p}{\partial r}+\mu\left(\frac{1}{r} \frac{\partial}{\partial r}\left(r \frac{\partial v_{r}}{\partial r}\right)-\frac{v_{r}}{r^{2}}+\frac{\partial^{2} v_{r}}{\partial x^{2}}\right)
\end{aligned}
$$

and momentum equations for the axisymmetric displacement $\mathbf{u}=\left[u_{r}, 0, u_{x}\right]$ of the compressible elastic wall of a circular tube with undisturbed radius $R_{0}$ (Nichols and O'Rourke, 2005)

$$
\begin{aligned}
& \rho_{s} \frac{\partial^{2} u_{x}}{\partial t^{2}}=\mu^{*}\left(\frac{1}{r} \frac{\partial}{\partial r}\left(r \frac{\partial u_{x}}{\partial r}\right)+\frac{\partial^{2} u_{x}}{\partial x^{2}}\right)+\left(\mu^{*}+\lambda^{*}\right) \frac{\partial \Delta}{\partial x} \\
& \rho_{s} \frac{\partial^{2} u_{r}}{\partial t^{2}}=\mu\left(\frac{1}{r} \frac{\partial}{\partial r}\left(r \frac{\partial u_{r}}{\partial r}\right)-\frac{u_{r}}{r^{2}}+\frac{\partial^{2} u_{r}}{\partial x^{2}}\right)+\left(\mu^{*}+\lambda^{*}\right) \frac{\partial \Delta}{\partial r} \\
& \Delta=\frac{1}{r} \frac{\partial\left(r u_{r}\right)}{\partial r}+\frac{\partial u_{x}}{\partial x}
\end{aligned}
$$

where $\lambda^{*}, \mu^{*}$ are Lame's coefficients, $\rho_{s}$ is wall density. 
Systems (3.6) and (3.7) are coupled via flow and stress continuity conditions at the fluid-solid interface $r=R_{0}$. The fastening conditions $(\mathbf{u}=\mathbf{0})$ or the unloading conditions $\left(\sigma_{n}=0\right)$ for deep and surface arteries, accordingly, can be applied at the outer surface $r=R_{0}+h_{0}$ of the tube, where $\sigma_{n}$ is the normal stress and $h_{0}$ is undisturbed wall thickness (Nichols and O'Rourke, 2005). When the pressure oscillation at the inlet of the tube is given as a Fourier series

$$
x=0: \quad P=\sum_{k=0}^{\infty} P_{k}^{0}(r) \mathrm{e}^{\mathrm{i} \omega_{k} t}
$$

the solution to (3.6) and (3.7) can also be found as a Fourier series for the variables $v_{r}, v_{x}, u_{r}$, $u_{x}, p$. The amplitudes of the corresponding waves can be found via the Bessel functions.

J.R. Womersley obtained the solution to (3.6)-(3.8) for an infinite tube. In the case of finite arteries, the pressure and flow continuity conditions at the outlet of the tube must be added in the form

$$
x=L: \quad \int_{0}^{R} r p d r=\pi R^{2} Z_{t} \int_{0}^{R} r v_{x} d r
$$

where $Z_{t}$ is the resistivity of terminal (downstream) vasculature, all coefficients of integration in the solution to (3.6), (3.7) can be found.

Later, model (3.6), (3.7) was generalized for circular tubes with non-constant radius $R_{0}(x)=\sqrt{S_{0}(x) / \pi}$, tethered tubes with corrected boundary conditions, and incompressible viscoelastic walls with different rheology (Nichols and O'Rourke, 2005; Kizilova et al., 2010). For the most popular Kelvin-Voigt model

$$
\tau_{s} \frac{\partial \sigma}{\partial t}+\sigma=E \varepsilon+\mu_{s} \frac{\partial \varepsilon}{\partial t}
$$

where $\varepsilon$ is strain, $\tau_{s}$ is stress relaxation time, $\mu_{s}$ is viscosity of the wall. Then the efficient Lame coefficient $\mu^{*}=\left(E+\mathrm{i} \omega \mu_{s}\right) /\left(1+\mathrm{i} \omega \tau_{s}\right)$ must be used in (3.7). For the Voigt model $\left(\tau_{s}=0\right)$, the generalized Lame coefficient is $\mu^{*}=E+\mathrm{i} \omega \mu_{s}$.

In (3.9), the simplest one-element model with resistivity $Z_{t}$ is used. A more general approach is based on the application of the 0D model (3.1) with two elements, i.e. 3- or 4-element Windkessel with double resistivity, compliance and inductance (Nichols and O'Rourke, 2005).

In the $2 \mathrm{D}$ case, the wave speed is a complex value

$$
c=c_{r}+\mathrm{i} c_{i}=c_{0} \sqrt{\frac{1-F_{01}}{1-\nu^{2}} \mathrm{e}^{\mathrm{i} \theta}} \quad c_{0}=\sqrt{\frac{E h}{2 \rho_{f} R}}
$$

where $c_{0}$ is determined by $(3.1), F_{01}=2 J_{1}(\delta) /\left(\delta J_{0}(\delta)\right)$ is the Womersley function, $J_{0}, J_{1}$ are the Bessel functions of the first kind and orders 0 and 1, accordingly, $\delta=\alpha \sqrt{\mathrm{i}^{3}}, \alpha=R \sqrt{\omega \rho_{f} / \mu_{f}}$ is the Womersley number, $\theta$ is the phase shift between the pressure and the wall displacement due to wall viscosity. For the Kelvin-Voigt rheological model (3.10) of the wall, one can easily compute

$$
\theta(\omega)=\tan \frac{\omega\left(\mu_{s}-E \tau_{s}\right)}{E+\omega^{2} \mu_{s} \tau_{s}}
$$

The resulting complex dependence $c(\omega)$ defined by (3.11) and (3.12) determines wave dispersion, i.e. dependence of the wave speed on the frequency. The value $c_{r}$ in (3.11) corresponds to the wave speed, while $c_{i}$ determines its spatial amplification rate. When model (3.6), (3.7) is applied to a complex network of tubes corresponding to a vascular system, the equations 
are separately solved for each tube and the unknown constants can be found from the pressure and flow continuity conditions at the bifurcations of the tubes. Usually, branching of the parent vessel with diameter $d_{0}$ into two $\left(d_{1}, d_{2}\right)$ of three $\left(d_{1}, d_{2}, d_{3}\right)$ daughter tubes is most common in vascular networks. When the characteristic wave admittances $Y_{j}$ of the tubes (Nichols and O'Rourke, 2005)

$$
Y_{j}=\frac{\pi d_{j}^{2}}{\rho_{f} c_{0}}
$$

are not matched, and $Y_{0} \neq Y_{1}+Y_{2}$ in the bifurcations or $Y_{0} \neq Y_{1}+Y_{2}+Y_{3}$ in the trifurcations, the reflected waves appear. Then the solution to (3.6), (3.7) can be found as a superposition of forward and reflected waves in the form (Nichols and O'Rourke, 2005)

$$
\begin{aligned}
& P^{(j)}\left(t, x_{j}\right)=P_{0}^{(j)}\left[\exp \left(\mathrm{i} \omega \frac{t-x_{j}}{c_{j}}\right)+\Gamma_{j} \exp \left(\mathrm{i} \omega \frac{t+\left(x_{j}-2 L_{j}\right)}{c_{j}}\right)\right] \\
& Q^{(j)}\left(t, x_{i}\right)=Y^{(j)} P_{0}^{(j)}\left[\exp \left(\mathrm{i} \omega \frac{t-x_{j}}{c_{j}}\right)-\Gamma_{j} \exp \left(\mathrm{i} \omega \frac{t+\left(x_{j}-2 L_{j}\right)}{c_{j}}\right)\right]
\end{aligned}
$$

where $j$ is the number of the tube, $P^{(j)}\left(t, x_{j}\right)$ and $Q^{(j)}\left(t, x_{i}\right)$ are pressures and volumetric flow rates, $P_{i}^{0}=\left.P_{i}\right|_{x_{i}=0}$ is the wave amplitude at the inlet of the tube, $x_{j} \in\left[0, L_{j}\right]$ is the longitudinal coordinate computed from the inlet of each tube with length $L_{j}, c_{j}$ is the wave speed determined by (3.11) for each tube, $\Gamma=p_{b} / p_{f}$ is the wave reflection coefficient, $p_{b}$ and $p_{f}$ are the amplitudes of the backward and forward waves.

According to (3.14), any positive wave reflection $(\Gamma>0)$ leads to an increase in the pressure amplitude and a decrease in the flow rate, because any reflected wave generates a retrograde flow (Kizilova, 2006). When $\Gamma<0$, the so called negative wave reflection is observed. Then the reflected wave propagates downstream and brings additional amount of fluid, which leads to a decrease in pressure at the outlet, increase in the pressure drop along the tube and, therefore, to an increase in the flow rate, which is known as the suction effect (Raphael et al., 2016). Negative wave reflections were found in the pulmonary circulation (Baksi et al., 2019), coronary vasculature (Hollander et al., 2001), along the thoracic aorta (Kizilova et al., 2019; Kizilova and Mizerski, 2018, and some others).

According to the Lighthill (1978) theory of wave reflection in the arteries

$$
\Gamma=\frac{Y_{0}^{(0)}-\sum_{j=1}^{m} Y_{0}^{(j)}}{Y_{0}^{(0)}+\sum_{j=1}^{m} Y_{0}^{(j)}}
$$

where the superscript indicates the number of the artery in the branching; 0 is for the parent artery, $j=1,2, \ldots, m$ are for daughter arteries; $m=2$ for bifurcations, $m=3$ for trifurcations.

It is important to note that for the complex wave velocity (3.11), (3.12), the wave admittance and reflection coefficients become complex values. Therefore, the complex reflection coefficient $\Gamma=\Gamma_{r}+\mathrm{i} \Gamma_{i}$ in (3.15) will contribute not only to the amplitude of the pressure and flow waves $\Gamma_{r}$, but also to the phase shift $\Gamma_{i}$ between the forward and reflected waves due to viscoelasticity of the wall.

\subsection{D fluid-structure interaction model}

The formulation of the general problem of blood circulation in the individual CT-based geometry is based on non-linear 3D Navier-Stokes equations for blood

$$
\nabla_{i} v_{i}=0 \quad \rho_{f}\left(\frac{\partial v_{i}}{\partial t}+v_{k} \nabla_{k} v_{i}\right)=-\nabla_{i} p+\mu_{f} \Delta v_{i}
$$

and mass and momentum balance equations for an incompressible viscoelastic wall 


$$
\begin{aligned}
& \nabla_{i} u_{i}=0 \quad \rho_{s} \frac{\partial^{2} u_{i}}{\partial t^{2}}=-\nabla_{i} p_{s}+\nabla_{k} \sigma_{i k} \\
& \tau_{s} \frac{\partial \sigma_{i}}{\partial t}+\sigma_{i}=A_{i k} \varepsilon_{k}+\mu_{s} \frac{\partial}{\partial t} \varepsilon_{i}
\end{aligned}
$$

where $A_{i k}$ is the matrix of elasticity coefficients, $\sigma_{i}$ and $\varepsilon_{i}$ are stress and strain vectors in the form, $a_{1-6}=\left\{a_{11}, a_{22}, a_{33}, a_{23}, a_{13}, a_{12}\right\}$, other variables are the same as in (3.6) and (3.7).

The 3D model (3.16)-(3.18) is usually solved by FEM for complex geometries restored from CT scans, MRI or other medical images (Salvi, 2017; Zenini et al., 2007). Due to the time consuming FEM computations, only truncated models with 1-10 vessels of arbitrary or realistic geometry can be computed, like the abdominal aorta with two renal and two iliac arteries, an 8-tube model of the aortic arch, coronary arteries, femoral artery, carotid arteries and others. In the most of the studied cases, the vessel walls were treated as rigid.

\section{Materials and methods}

Geometry of arterial systems is usually described by sets of diameters, lengths, branching angles, wall properties $\left\{L_{j}, d_{j}, \alpha_{j}, h_{j}, E_{j}, \nu_{j}, \theta_{j}\right\}_{j=1}^{N}$ and topology of the vasculature (connectivity matrix). In this paper, regularities of wave propagation along the most complete model of the human aorta with all existing side branches is considered. The considered model is composed of 36 aortic segments and 57 side branches (Fig. 2); the former are feeding arteries of the inner organs like heart $(\mathrm{N} 1,2)$, spleen (N16), liver (N17), kidneys (N21,22), intestine (N18,26), whereas the others supply glands, muscles and other tissues. Most of the arteries feeding the respiratory system and vertebral column are absent in the common 55-tube model. As it was mentioned above, only one intercostal artery (N4 in Fig. 1) is included in the 55-tube model while actually there are 9 pairs of intercostal and 13 more pairs of other medium arteries (Fig. 2). They produce a small but continuous contribution to the aortic conductivity, pressure and flow distributions (Kizilova et al., 2019; Kizilova and Mizerski, 2018). Due to their synergy in the negative wave reflection, the resulting influence may be essential. It was shown, thanks to their presence, that the blood flow accelerates due to the suction effect.

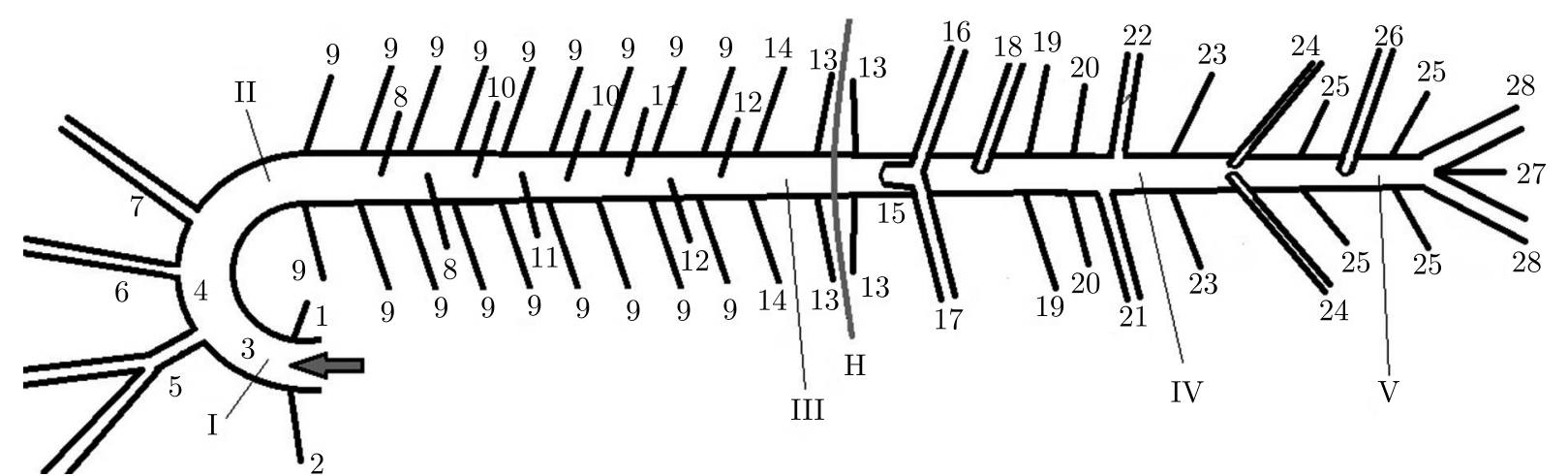

Fig. 2. Model of the aorta: left (1) and right (2) coronary, aortic root (3) and arch (4), brachiocephalic trunk (5), left carotid (6), subclavian (7), bronchial (8), intercostal (9), esophageal (10),

mediastinal (11), pericardial (12), diaphragmal (13), subcostal (14), celiac trunk (15), splenic (16),

hepatic (17), upper mesenchymal (18), suprarenal (19), upper lumbar (20), left (21) and right (22) renal, lumbar (23), testicular (24), lower lumbar (25), lower mesenchymal (26), sacral (27) and iliac (28) arteries; the arrow indicates blood inflow; $H$ - diaphragm, I-VII - measurement sites

The morphometric data used in this study have been measured on five cadavers during post-mortem examination (Kizilova et al., 2019; Kizilova and Mizerski, 2018). The $P(t)$ and $V(t)$ waveforms in different locations along the aorta have been measured on ten healthy volunteers 
(ages from 18 to 42) by triplex scanner "Sonolaine Elegra Advanced" (Siemens) (Fig. 3a). The corresponding locations are marked in Fig. 2 as I (for Fig. 3a) and II (for Fig. 3b). The $P(t)$ and $V(t)$ curves in the main coronary arteries have been measured by transesophageal ultrasound. The measured curves have been smoothed by the Bayesian filter and analyzed. In total, 150 pairs of $P(t)$ and $V(t)$ curves have been recorded (30 measurement sites per an individual) and used for validation of the individual 93-tube model of aorta (Kizilova and Mizerski, 2018). The detailed measurement data for the five individuals and the averaged values are reported in (Kizilova et al., 2019; Kizilova and Mizerski, 2018). Due to different body height of the individuals, their geometric models have been normalized by the total length along the aorta from the aortic valve to the aortic bifurcation. The data on relative locations of the branches along the aorta are presented in Table 1 in comparison to the similar 55-tube tree data given in (Wang and Parker, 2004). As it is clear from Table 1, the geometric datasets are quite different and exhibit individual scatter in lengths of the aortic segments that can influence local travel times of the incident and reflected waves in each segment and along the entire aorta. The corresponding distributions of wave speeds and travel times are presented in Fig. 4a,b respectively. The wave speeds have been computed on morphometric data (Kizilova et al., 2019; Kizilova and Mizerski, 2018) by (3.11), (3.12) or taken from the tables computed in (Wang and Parker, 2004; Epstein et al., 2015). The curves represent physically known dependence of the pulse wave velocity on the diameter of the blood vessel or (in experiments) latex tube (Nichols and O'Rourke, 2005; Hughes et al., 2013), as it is also clearly visible from (3.11). The wave speed in the fluid-filled elastic tubes increases while the diameter decreases. Since the aorta is a tapered viscoelastic tube, the wave speed non-linearly increases along it, which is responsible for pulse wave peaking and steeping, which are well known effects in arteries (Nichols and O'Rourke, 2005; Salvi, 2017). The lengths of aorta are scaled to 1 , and it is evident that the data computed on our datasets for five individuals well correspond to the recent data from (Epstein et al., 2015). At the distances $x 17-19 \%$ and $x 70 \%$ of $L_{a}$, rapid increases in the wave speed determined by sudden physiological narrowing of the aorta are present. The earlier data (Wang and Parker, 2004) do not demonstrate such a clear dependence but also exhibit non-linear behavior. The corresponding experimental dependencies $c(x)$ (Salvi, 2017; Hughes et al., 2013) are smoother because of the smaller number of locations (3.4)-(3.6) and more space between them for smooth approximation.
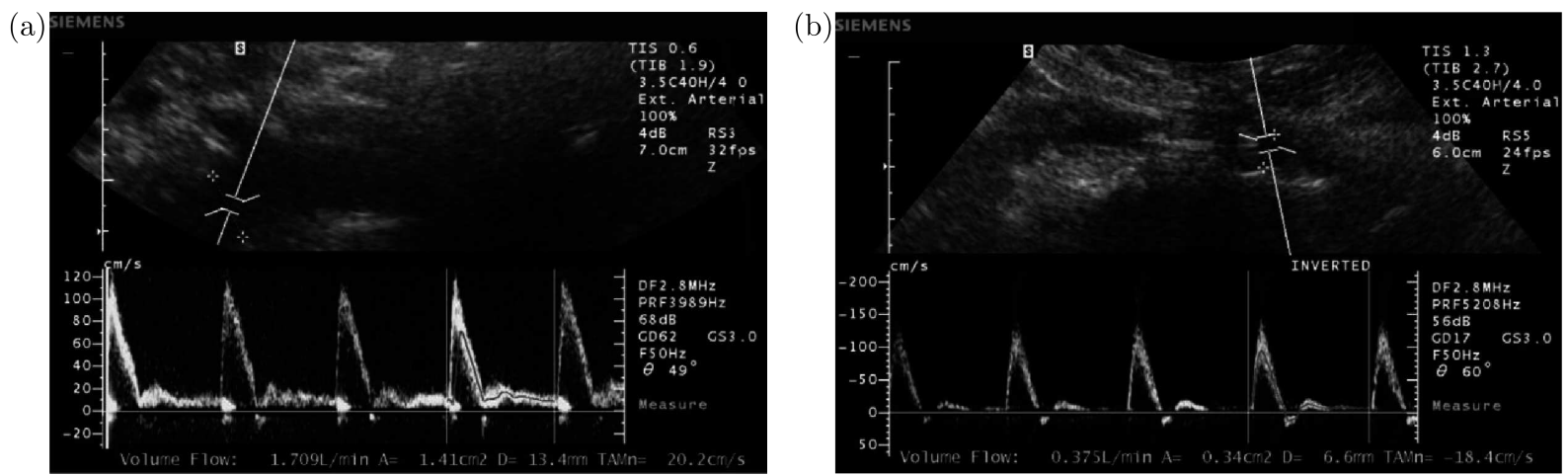

Fig. 3. Measured flow rate curves $Q(t)$ at locations III (a) and V (b) in Fig. 2

\section{Numerical procedure}

The material parameters and approximations for the wall thickness and Young's modulus and the same as in (Kizilova and Mizerski, 2018) and correspond to the parameters of the healthy blood and aortic wall. 
Table 1. Relative locations of the side branching along the aortic length scaled to 1

\begin{tabular}{|l|c|c|c|c|}
\hline \multirow{2}{*}{ Artery } & \multicolumn{4}{|c|}{ Relative distance } \\
\cline { 2 - 5 } & {$[13],[15]$} & {$[11]$} & {$[28]$} & {$[31]$} \\
\hline \hline Aortic valve & 0 & 0 & 0 & 0 \\
\hline Coronary & $0.0043 \pm 0.014$ & - & - & - \\
\hline Brachiocephalic & $0.1333 \pm 0.008$ & $0.041 \pm 0.0098$ & 0.09009 & 0.103943 \\
\hline Left common carotid & $0.1660 \pm 0.009$ & $0.075 \pm 0.011$ & 0.135135 & 0.145161 \\
\hline Left subclavian & $0.1858 \pm 0.013$ & $0.12 \pm 0.011$ & 0.222973 & 0.225806 \\
\hline 1st pair of intercostals & $0.1872 \pm 0.007$ & $0.20 \pm 0.013$ & - & - \\
\hline 2nd & $0.1901 \pm 0.015$ & $0.24 \pm 0.014$ & - & - \\
\hline Bronchial & $0.2496 \pm 0.01$ & - & - & - \\
\hline 3rd & $0.2950 \pm 0.01$ & $0.29 \pm 0.0066$ & - & - \\
\hline Bronchial & $0.3404 \pm 0.009$ & - & - & - \\
\hline 4th & $0.3858 \pm 0.01$ & $0.34 \pm 0.0087$ & - & - \\
\hline Esophageal & $0.4015 \pm 0.008$ & - & - & - \\
\hline 5th & $0.4312 \pm 0.01$ & $0.38 \pm 0.0080$ & 0.34009 & 0.333333 \\
\hline Mediastinal & $0.4525 \pm 0.006$ & - & - & - \\
\hline 6th & $0.4766 \pm 0.013$ & $0.42 \pm 0.0064$ & - & - \\
\hline Esophageal & $0.4994 \pm 0.008$ & - & - & - \\
\hline 7th & $0.5220 \pm 0.014$ & $0.47 \pm 0.0087$ & - & - \\
\hline Mediastinal & $0.5327 \pm 0.009$ & - & - & - \\
\hline 8th & $0.5674 \pm 0.01$ & $0.51 \pm 0.0096$ & - & - \\
\hline Pericardial & $0.5923 \pm 0.015$ & - & - & - \\
\hline 9th & $0.6128 \pm 0.007$ & $0.55 \pm 0.012$ & - & - \\
\hline Pericardial & $0.6582 \pm 0.01$ & - & - & - \\
\hline Subcostal artery & $0.6837 \pm 0.011$ & $0.61 \pm 0.012$ & - & - \\
\hline Diaphragmal & $0.7121 \pm 0.0065$ & - & - & - \\
\hline Celiac trunk & $0.7248 \pm 0.007$ & $0.69 \pm 0.013$ & 0.574324 & 0.548387 \\
\hline Upper mesenchymal & $0.7716 \pm 0.008$ & - & 0.693694 & 0.657706 \\
\hline Suprarenal & $0.7730 \pm 0.01$ & - & - & - \\
\hline Upper lumbar & $0.7745 \pm 0.015$ & - & - & - \\
\hline Right renal artery & $0.7751 \pm 0.013$ & $0.73 \pm 0.012$ & 0.716216 & 0.698925 \\
\hline Left renal artery & $0.7759 \pm 0.007$ & $0.78 \pm 0.014$ & 0.738739 & 0.740143 \\
\hline Lumbar & $0.8043 \pm 0.01$ & - & - & - \\
\hline Testicular & $0.8170 \pm 0.009$ & - & - & - \\
\hline Lower lumbar & $0.8752 \pm 0.008$ & - & - & - \\
\hline Lower mesenchymal & $0.9007 \pm 0.011$ & - & 0.977477 & 0.958781 \\
\hline Lower lumbar & $0.9333 \pm 0.007$ & $0.86 \pm 0.015$ & - & - \\
\hline Aortic bifurcation & 1 & 1 & 1 & 1 \\
\hline 113 - Kitova and & & - & - & - \\
\hline
\end{tabular}

[13] - Kizilova and Mizerski (2018), [15] - Kizilova et al. (2019)

[11] - Kassa (2006), [28] - Wang and Parker (2004), [31] - Willemet et al. (2015)

The complex resistivity of the terminal 0D elements for each of the 57 side branching tubes have been computed on the known geometries of the corresponding vasculatures based on the parallel and serial addition of the connected tubes as it is explained in (Epstein et al., 2015). In (Kizilova and Mizerski, 2018), a method based on consecutive corrections of the parameters of the structured tree outflow boundary conditions was tested and validated based on in vivo measurement data. It was found that any small variations in the branching constants $\varphi_{j}, \psi_{j}$ that 


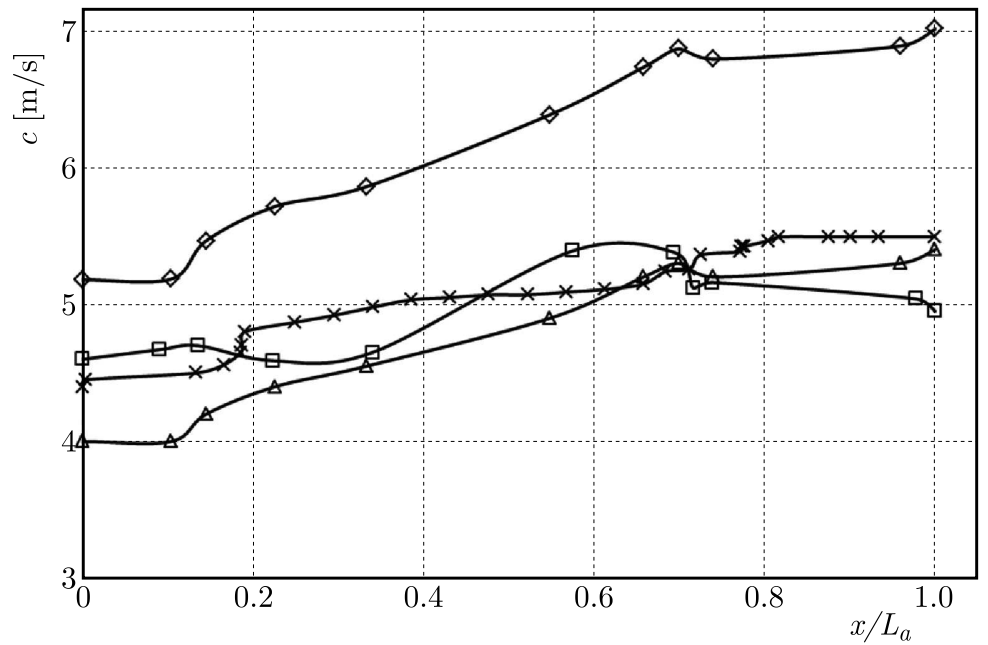

Fig. 4. Wave speed $c[\mathrm{~m} / \mathrm{s}]$ distribution along the aorta according to (Sun et al., 2000; Hollander et al.,

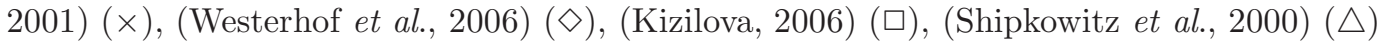

determine the structured outflow trees $j=1,2, \ldots, N$ influenced the pressure and flow oscillations in the parent tube only, wheras the signals in other tubes remained almost unperturbed (Kizilova and Mizerski, 2018). It may be connected to fast attenuation of the reflected waves and small input of the multiple reflections (Nichols and O'Rourke, 2005). In that way, when the inlet pressure profile was assigned in form (3.8), the first iteration of $P(t)$ and $V(t)$ computations in sites I, II and others along the aorta exhibited noticeable differences with the measured signals (Fig. 3) when the initial values $\varphi_{j}=1, \psi_{j}=1$ were used. When the calibration procedure for $\varphi_{j}, \psi_{j}$ values was conducted by the least square method, the computed curves became quite close to the measured signals (Kizilova and Mizerski, 2018). In that way, the structured tree outflow boundary conditions at the terminuses provided a better approximation giving different wave impedances for different harmonics.

The signal $P(t)$ at the inlet of the aorta typical for healthy humans (Nichols and O'Rourke, 2005; Epstein et al., 2015) has been taken in form (3.8) as a superposition of $k=8$ harmonics. The pressure and flow waveforms have been computed in the middle cross section of each segment in the aortic model (Fig. 2), and the corresponding dependencies measured in vivo at locations I-VII have been used for identification of individual parameters. The digitized signals (Fig. 3) have also been presented as Fourier series (3.8) and compared to the corresponding harmonics of the computed signals. The frequency in (3.8) has been determined by the individual heart beat rate taken from the ultrasound data. When the model parameters had been identified, the model was used for studying the influence of wall rigidity, wave reflection coefficients, geometry, as well as model sensitivity to the parameters. Some of the results were reported in (Kizilova et al., 2019; Kizilova and Mizerski, 2018).

\section{Results and discussions}

\subsection{Model sensitivity}

The model was found quite sensitive to wave reflection coefficients at certain locations crucial for the wave reflection like at the level of cervical, kidney and iliac arteries. The $P(t)$ and $V(t)$ waveforms at fixed relative locations $x / L=0.05,0.25,0.5,0.75,0.95$ along the aorta are presented in Fig. 5. The corresponding branches and wave speeds at their location can be found in Table 1 and Fig. 4, respectively. When the resistivity of iliac arteries is twice higher than the measured undisturbed individual values because of iliac stenosis or occlusion, the blood flow 

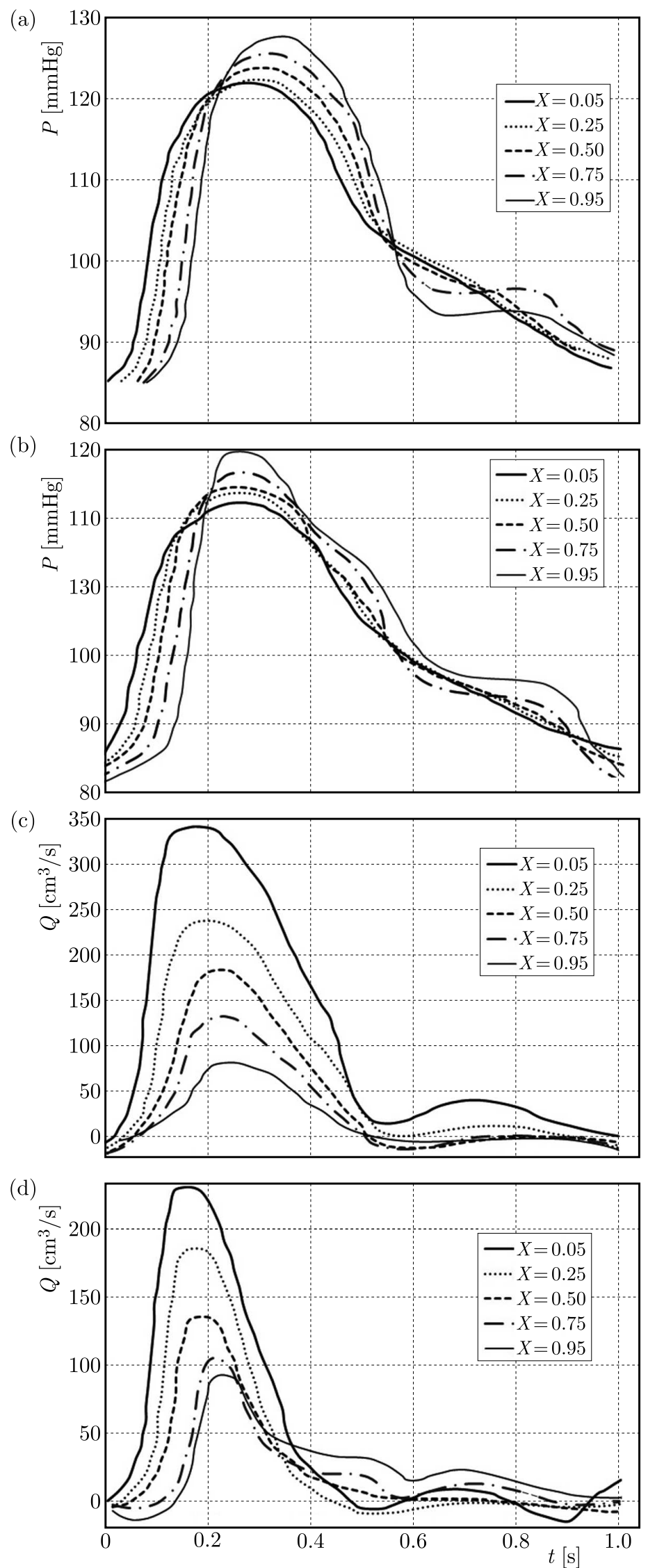

Fig. 5. Pressure $P(t)-(\mathrm{a})$, (b) and flow rate $Q(t)-(\mathrm{c})$, (d) distributions at different relative distances along the aorta for low (a), (c) and high (b), (d) resistivity of iliac arteries 
becomes noticeably lower (Fig. 5d) in comparison to the unaffected values (Fig. 5c) at almost the same systolic and diastolic pressures (Fig. 5a,b). It means that the blood outflow to the side branches remains high but the low extremities suffer of blood insufficiency, and the blood inflow to them is $\sim 1.8$ lower than its unaffected value (Fig. $5 \mathrm{c}, \mathrm{d})$. In healthy vessels $\left(E_{0}=10^{5}-10^{6} \mathrm{~Pa}\right)$, the pressure wave attenuation and steepening along the artery is ehxibited, and a moderate dicrotic wave in the diastolic part of the wave appears and develops (Fig. 5a). Changes in the maximal pressure along the aorta are moderate provided that the wave reflection coefficients correspond to the measured (or conventionally healthy) values. Therefore, due to small negative wave reflection along the thoracic aorta (Kizilova and Mizerski, 2018) and the suction effect, the dicrotic wave is absent along this path (Fig. 5a) and the flow rate is high with clear peak values during the systole (Fig. 5b). Similar steepening and a higher dicrotic wave due to wave reflection at the level of aortic bifurcation is observed in the case of high iliac resistivity (Fig. 5b). The obtained results are in correspondence to the recent measurement data on the flow and pressure waves in the aorta at different reflection coefficients of the iliac arteries (N28, 29 in Fig. 1).

\subsection{Reverse blood flow at the end of systole}

Reverse aortic blood flow at the end of systole is proper to human blood circulation. In healthy patients, it has a short duration, whereas in the case of positive wave reflection coefficients in the abdominal aorta (Kizilova and Mizerski, 2018), the wave reflection contributes to a longer reverse flow and a lower blood flow rate to the mesenteric and other downstream arteries. In in vivo conditions, the active vasodilatation/constriction reactions of the side vessels can influence the blood distribution between the organs according to their demands. As it is observed in the in vitro and in vivo databases, the scatter in the wave reflection coefficients is always higher in a live patients (Kizilova et al., 2019; Hollander et al., 2001) because their arteries are always physiologically constricted, whereas in the post-mortem state all arteries are relaxed. Anyway, when the narrowing of some vessels is produced by stenosis, occlusion or other pathological reasons, the autoregulatory reactions might be insufficient to maintain the needed blood distribution along the aorta affected by the reverse flow at the end of systole (Salvi, 2017; Hughes et al., 2013).

According to the results presented in Fig. 5c,d, the duration of the reverse flow $(V<0)$ depends on the location along the aorta and the resistivity (i.e. wave reflection coefficient) at the iliac arteries. The magnitude and duration of the reverse flow decreases along the thoracic aorta and then starts to increase along the abdominal aorta, which corresponds to the measurement results from the 55-tube tree (Wang and Parker, 2004) and numerous experimental data (Nichols and O'Rourke, 2005; Salvi, 2017).

\subsection{The aortic length must be scaled to 1 for correct analysis of the experimental data}

The data on arterial diameters are more uniform than on lengths, which produces significant differences in phases of the waves reflected at the same branches in different individuals. For instance, the aortic length between the inlet and aortic bifurcations varied within $35-72 \mathrm{~cm}$. When the individual dimensions are scaled by the personal height, and then averaged and scaled to a fixed height, it produces fewer differences between personal specific coordinates of the same branches along the aorta (see Table 1). The simulation results obtained on the shorter aortic model $L_{a}=33.5 \mathrm{~cm}$ (Epstein et al., 2015) exhibit early return of the reflected wave when the arterial wall has the same viscoelastic properties in both short and long $L_{a}=70.5 \mathrm{~cm}$ (Hollander et al., 2001) aortic models and, therefore, the wave speeds along the aorta are of the same values (Fig. 4). Then the reflected wave is located closer to the pressure peak (Fig. 6a) than in the longer aortic model (Fig. 5a,b). The blood velocity waveform demonstrates deeper and longer negative peak at the end of the systole (Fig. 6b) than those in the longer aorta (Fig. 5c,d). Therefore, 
individual variations in geometry and geometry based wave reflection coefficients play more essential role on the pulse wave dynamics along the aorta than other model parameters. When the aortic length is scaled to 1 , a clear regularity in the location of the sites of high positive, zero and negative wave reflections appears. An example of the distribution of such locations can be found in (Kizilova et al., 2019; Kizilova and Mizerski, 2018).

(a)

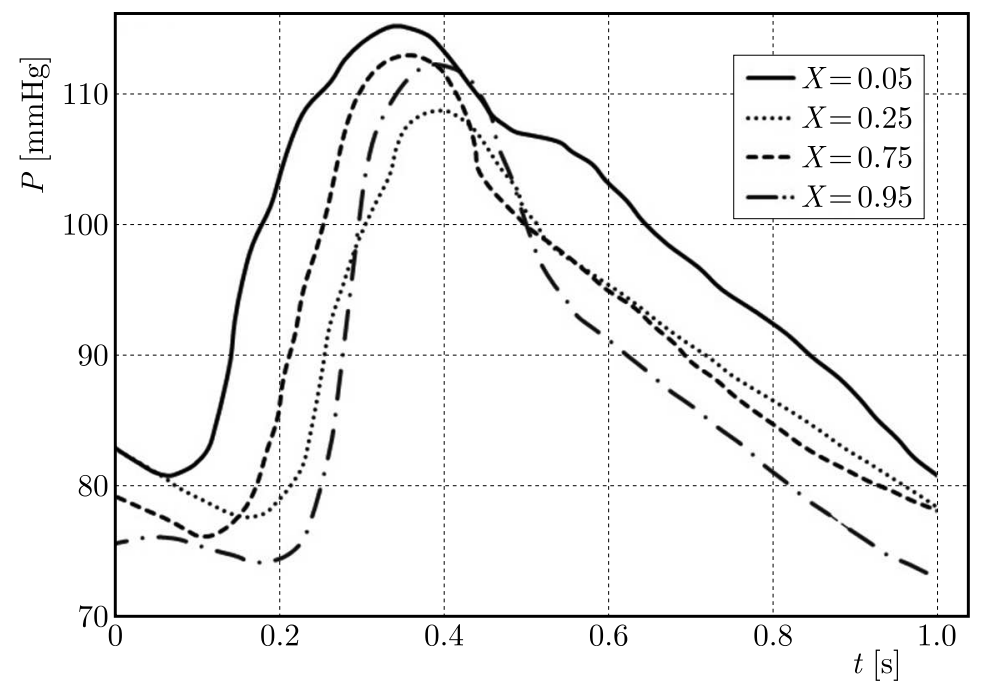

(b)

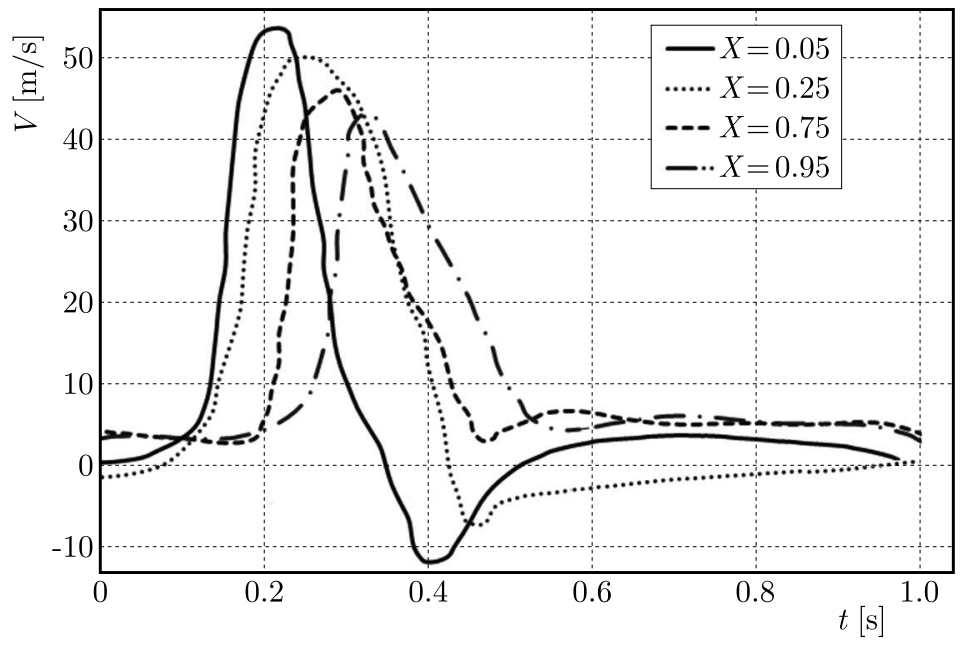

Fig. 6. Distributions of the $V(t)(\mathrm{a})$ and $P(t)$ (b) waveforms along short healthy aorta

\subsection{Model validation}

Since the blood pressure and flow could be measured on volunteers only at a restricted number of locations, mostly over the larger side branches and the thoracic aorta were unavailable in the US. The direct validation of the proposed aortic model is possible on special invasive catheter measurements that will be done in future. In this paper, the existing data on evolution of pressure and flow waves measured along aorta (1), our measurements on ten volunteers at seven locations along aorta (2) as well as the patterns computed on the 55-tube model in literature (3) are used for the model validation. The results are presented in Fig. 7. Thick black lines correspond to typical flow waves proper to healthy arteries, which have been digitized from (Salvi, 2017). Since the curves have been measured on individuals with different heights, they have been rescaled to the average height over the database $(171 \mathrm{~cm})$. The dots correspond to the US measured curves on ten healthy volunteers. For comparison reasons, the average height of the group $(173.5 \mathrm{~cm})$ has been rescaled to $171 \mathrm{~cm}$. The same rescaling has been done for the wave period (i.e. heart 
rate). Grey dashed lines correspond to the curves computed on the 93-tube model with the model parameters identified from the averaged geometric model by the procedure described in (Kizilova and Mizerski, 2018). Dotted lines have been rescaled from the numerical computations on the 55-tube model (Wang and Parker, 2004). Qualitative differences with the last set of curves may be explained by the inlet boundary conditions. In (Wang and Parker, 2004), the computations were done for the sinusoidal type of flow oscillations in the aortic root, whereas in the rest of curves the inlet flow corresponded to the realistic one that can be found in (Kizilova and Mizerski, 2018) (curve I in Fig. 3) or in (Salvi, 2017).

(a)

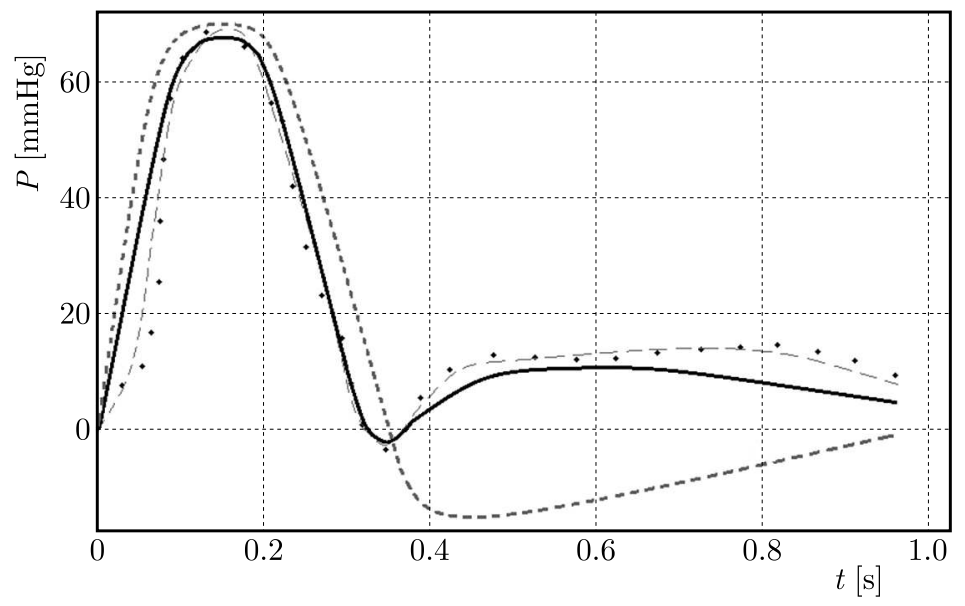

(b)

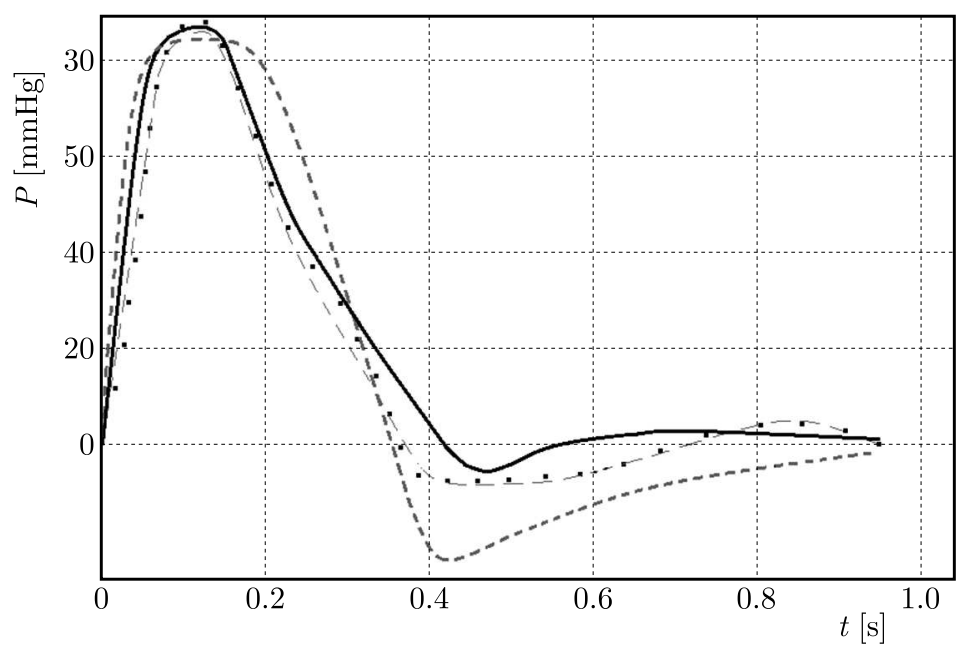

Fig. 7. Flow waves $V(t)$ at locations II (a), V (b), VI (c) (Fig. 1) along the aorta; experimental curves from (Salvi, 2017) (solid lines), US measurements (dots), numerical computations on the developed model (dashed lines) and on the 55-tube model (dotted lines)

As one can see, the developed 93-tube model of human aorta provides quite good qualitative and quantitative correspondence to the in vivo measured signals and previous computations on the simpler 21-tube model of aorta. In the case of healthy arteries, the pulse wave generated by the heart propagates along the thoracic aorta at a relatively low wave speed (Fig. 4) and late reflected waves producing small dicrotic waves at the end of diastole only. Due to smaller diameter of the thoracic aorta and, therefore higher wave speed, the pulse wave accelerates and the reflected waves contribute to its early diastolic part and peak, which produces well-known wave peaking and steeping (Nichols and O'Rourke, 2005). That is why the wave amplitude gradually decreases during the thoracic aorta and then again increases due to the peaking along the abdominal aorta. Presence of the negative reflection sites along the thoracic aorta could accelerate the flow but due to the low wave speed it will not produce noticeable peaking. 
After the validation, the model can be used for determination of individual parameters for patient-specific cardiovascular models and further modeling of the outcomes of surgical and therapeutic procedures.

\section{Conclusions}

The most detailed among the existing biomechanical models of human aorta, a 93-tube model consisting of 36 aortic segments and 57 side branches is developed based on post mortem measurements on 5 individuals. Statistical regularities in the branching and reflection coefficients studied in (Kizilova et al., 2019; Kizilova and Mizerski, 2018) showed that aorta can be considered as an optimal waveguide providing non zero wave reflections as it was shown on the simplified 21-tube model (10 aortic segments and 11 side branches) but negative wave reflections which produced accelerated blood flow due to well-knows suction phenomena. A complex mathematical model of the aorta based on the 1D and linearized 2D models of blood flow in the larger arteries, 0D models for the terminal elements at the open end of the tubes has been developed. The areas of specific interest for doctors like stenosis, coarctation, aneurism and others can be modeled by the 3D FSI model. The model has been validated on the in vivo measurement data (Kizilova and Mizerski, 2018). In this study, further numerical computations on the model aimed at estimation of the model sensitivity to its parameters, regularities of the pressure and flow wave evolution along the aorta at different wave reflection conditions have been conducted.

The physically consistent pressure wave peaking and steepening along the aorta, blood flow decrease in the amplitude, negative flow at the end systole, and dicrotic wave appearance and development due to numerous wave reflections have been obtained. It is shown that the individual geometry plays an essential role in the location of positive and negative wave reflection sites along the aorta and, thus, in the pressure and flow patterns as well as blood distribution into the side branches. High sensitivity of the flow evolution along the aorta on the wave reflection coefficients is confirmed. The numerical results show that the amplitude of pressure waves increases distally, whereas the amplitude of velocity decreases in the abdominal aorta and then begins to increase. The magnitude and duration of the reverse flow with negative velocity decreases and then begins to increase in the abdominal aorta.

The model is validated by a comparative study of the same dependencies computed previously on the 55-tube model and obtained from measurements. It can be used for determination of individual parameters for patient-specific cardiovascular models and further modeling of the outcomes of the surgical and therapeutic procedures.

Acknowledgments

The authors are grateful to the organizers and participants of the XXIII Fluid Mechanics Conference KKMP2018 (Zawiercie, Poland, 9-12 September 2018) for their interest to the topic and stimulating discussions.

\section{References}

1. Alastruey J., Parker K.H., Peiró J., Byrd S.M., Sherwin S.J., 2007, Modelling the circle of Willis to assess the effects of anatomical variations and occlusions on cerebral flows, Journal of Biomechanics, 40, 1794-1805, DOI: 10.1016/j.jbiomech.2006.07.008

2. Alastruey J., Parker K.H., Peiró J., Sherwin S.J., 2006, Can the modified Allen's test always detect sufficient collateral flow in the hand? A computational study, Computer Methods in Biomechanics and Biomedical Engineering, 9, 353-361 
3. Baksi A.J., Davies J.E., Hadjiloizou N., Baruah R., Unsworth B., Foale R.A., Korolkova O., Siggers J.H., Francis D.P., Mayet J., Parker K.H., Hughes A.D., 2019, Attenuation of reflected waves in man during retrograde propagation from femoral artery to proximal aorta, International Journal of Cardiology, 202, 441-445, DOI: 10.1016/j.ijcard.2015.09.064

4. Díaz-Zuccarini V., Narracott A.J., Burriesci G., Zervides C., Rafiroiu D., Jones B., Hose D.R., LAwford P.V., 2006, Adaptation and development of software simulation methodologies for cardiovascular engineering: present and future challenges from an end-user perspective, Philosophical Transactions of the Royal Society A, 367, 2655-2666, DOI: 10.1098/rsta.2009.0052

5. Epstein S., Willemet M., Chowienczyk P.J., Alastruey J., 2015, Reducing the number of parameters in 1D arterial blood flow modeling: less is more for patient-specific simulations, American Journal of Physiology, 309, H222-H234

6. Formaggia L., Gerbeau J.-F., Nobile F., Quarteroni A., 2001, On the coupling of 3D and 1D Navier-Stokes equations for flow problems in compliant vessels, Computer Methods in Applied Mechanics and Engineering, 191, 561-582

7. HALl D.C., BA LE Q., 2015, Monitoring and evaluation of one health projects; lessons from Southeast Asia, Procedia - Social and Behavioral Sciences, 186, 681-683, DOI: 10.1016/j.sbspro.2015.04.070

8. Hollander E.H., Wang J.J., Dobson G.M., Parker K.H., Tyberg J.V., 2001, Negative wave reflections in pulmonary arteries, American Journal of Physiology, 281, 895-902, DOI: 10.1152/ajpheart.2001.281.2.H895

9. Hughes A.D., Davies J.E., PARker K.H., 2013, The importance of wave reflection: a comparison of wave intensity analysis and separation of pressure into forward and backward components, Conference of the IEEE Engineering in Medicine and Biology Society, 229-232

10. Karamanoglu M., Gallagher D.E., Avolio A.P., O’Rourke M.F., 1995, Pressure wave propagation in a multibranched model of the human upper limb, American Journal of Physiology, 269, H1363-H1369

11. Kassab G.S., 2006, Biomechanics of the cardiovascular system: the aorta as an illustratory example, Journal of the Royal Society Interface, 3, 719-740

12. Kizilova N.N., 2006, Pressure wave propagation in liquid-filled tubes of viscoelastic material, Fluid Dymanics, 41, 434-446

13. Kizilova N., Mizerski J., 2018, Validation of numerical models for flow simulation and wave propagation along human aorta, Journal of Physics: Conference Series, 1101, 012014

14. Kizilova N., Philippova H., Zenin O., 2010, A realistic model of human arterial system: blood flow distribution, pulse wave propagation and modeling of pathology, [In:] Mechanika w Medycynie, Korzynski M., Cwanka J. (Edit.), 10, Rzeszow University Press, 103-118

15. Kizilova N., Solovyova H., Mizerski J., 2019, Modeling of pulse wave propagation and reflection along human aorta, [In:] Biomechanics in Medicine and Biology, K. Arkusz, R.Będziński, T. Klekiel, S. Piszczatowski (Edit.), Springer Series "Advances in Intelligent Systems and Computing", 831, 23-35

16. Lighthill M.J., 1978, Waves in Fluids, Cambridge: Cambridge University Press

17. Michail M., Davies J.E.R., Cameron J.D., Parker K.H., Brown A.J., 2018, Pathophysiological coronary and microcirculatory flow alterations in aortic stenosis, Nature Reviews Cardiology, 15, 420-431, DOI: 10.1038/s41569-018-0011-2

18. Narayan O., Parker K.H., Davies J.E., Hughes A.D., Meredith I.T., Cameron1 J.D., 2017, Reservoir pressure analysis of aortic blood pressure: An in-vivo study at five locations in humans, Journal of Hypertension, 35, 2025-2033, DOI: 10.1097/HJH.0000000000001424

19. Negoita M., Hughes A.D., Parker K.H., Khir A.W., 2017, Non-invasive technique for determining local pulse wave velocity in humans ascending aorta, Computing in Cardiology, 44, 1-4 
20. Nichols W., O'Rourke M., 2005, McDonald's, Blood Flow in Arteries. Theoretical, Experimental and Clinical Principles, Oxford: Oxford University Press

21. Quarteroni A., 2001, Modeling the Cardiovascular System: A Mathematical Challenge, Mathematics Unlimited and Beyond, Springer, Berlin, 961-970

22. Quarteroni A., Tuveri M., Veneziani A., 2000, Computational vascular fluid dynamics: Problems, models and methods, Computer Visualization Sciences, 2, 163-197

23. Raphael C.E., Cooper R., Parker K.H., Collinson J., Vassiliou V., Pennell D.J., De Silva R., Hsu L.Y., Greve A.M., NijJer S., Broyd C., Ali A., Keegan J., Francis D.P., Davies J.E., Hughes A.D., Arai A., Frenneaux M., Stables R.H., Di Mario C., Prasad S.K., 2016, Mechanisms of myocardial ischemia in hypertrophic cardiomyopathy. Insights from wave intensity analysis and magnetic resonance, Journal of American College of Cardiology, 68, 1651-1660, DOI: 10.1016/j.jacc.2016.07.751

24. Salvi P., 2017, Pulse Waves. How Vascular Hemodynamics Affects Blood Pressure, 2nd ed. Springer

25. Shipkowitz T., Rodgers V.G.J., Frazin L.J., Chandran K.B., 2000, Numerical study on the effect of secondary flow in the human aorta on local shear stresses in abdominal aortic branches, Journal of Biomechanics, 33, 717-728

26. Sun Y.-H., Anderson T.J., PArker K.H., Tyberg J.V., 2000, Wave-intensity analysis: a new approach to coronary hemodynamic, Journal of Applied Physiology, 89, 1636-1644

27. Takach T., Reul G., Cooley D., Duncan J.M., Livesay J.J., Ottet D.A., 2006, Myocardial thievery: the coronary-subclavian steal syndrome, Annals of Thoracic Surgery, 81, 386-392, DOI: $10.1016 /$ j.athoracsur.2005.05.071

28. WAng J.J., PARker K.H., 2004, Wave propagation in a model of the arterial circulation, Journal of Biomechanics, 37, 4, 457-470. DOI: 10.1016/j.jbiomech.2003.09.007

29. Westerhof N., Bosman F., de Vries C.J., Noordergraaf A., 1969, Analog studies of the human systemic arterial tree, Journal of Biomechanics, 2, 121-143

30. Westerhof B.E., Guelen I., Westerhof N., Karemaker J.M., Avolioet A., 2006, Quantification of wave reflection in the human aorta from pressure alone: a proof of principle, Hypertension, 48, 4, 595-601, DOI: 10.1161/01.HYP.0000238330.08894.17

31. Willemet M., Chowienczyk P., Alastruey J., 2015, A database of virtual healthy subjects to assess the accuracy of foot-to-foot pulse wave velocities for estimation of aortic stiffness, American Journal of Physiology, 309, H663-H675

32. Zenin O.K., Kizilova N.N., Filippova E.N., 2007, Studies on the structure of human coronary vasculature, Biophysics, 52, 499-503 